\title{
Assessing the Expected Accuracy of Probe VeHicle TRAVEL TIME REPORTS
}

\author{
By Bruce Hellinga P.Eng., ${ }^{1}$ and Liping $\mathrm{Fu}^{2}$
}

\section{Abstract:}

The use of probe vehicles to provide estimates of link travel times has been suggested as a means of obtaining travel times within signalized networks for use in advanced traveler information systems (ATIS). Past research in the literature has provided contradictory conclusions regarding the expected accuracy of these probe based estimates, and consequently has estimated different levels of market penetration of probe vehicles required to sustain accurate data within an ATIS. This paper examines the effect of sampling bias on the accuracy of the probe estimates. An analytical expression is derived on the basis of queuing theory to prove that bias in arrival time distributions and/or in the proportion of probes associated with each link departure turning movement, will lead to a systematic bias in the sample estimate of the mean delay. Subsequently, the potential for and impact of sampling bias on a signalized link is examined by simulating an arterial corridor. The analytical derivation and the simulation analysis show that the reliability of probe based average link travel times is highly affected by sampling bias. Furthermore, this analysis shows that the contradictory conclusions of previous research are directly related to the presence or absence of sample bias.

Key Words: sampling, probe vehicles, traffic information, bias, monitoring

\footnotetext{
${ }^{1}$ Assistant Professor, Department of Civil Engineering, University of Waterloo, Waterloo, Ontario, Canada N2L 3G1 Email: bhellinga@uwaterloo.ca

${ }^{2}$ Assistant Professor, Department of Civil Engineering, University of Waterloo, Waterloo, Ontario, Canada N2L 3G1 Email: lfu@uwaterloo.ca
} 


\section{Introduction:}

The successful wide scale deployment of Advanced Traveler Information Systems (ATIS) and Advanced Traffic Management Systems (ATMS) depends on the ability to obtain and subsequently disseminate information that accurately reflects network traffic conditions. Many different techniques for assessing traffic conditions have been proposed. However, one method in particular, namely the use of vehicles that are capable of transmitting link travel times to the traffic management center, has received considerable attention. The use of probe vehicles enables a sample of the travel times experienced by all vehicles traversing the link to be obtained. This paper examines the use of probe vehicles on signalized links and addresses the critical question of "How accurately do the probe vehicle travel times (sample) reflect the travel times of all the vehicles (population) that traversed the link?"

A number of researchers have previously investigated the expected reliability of probe travel time reports. Van Aerde et al (1993) developed an analytical expression for the reliability of probe travel times for signalized links and verified these expressions using simulated data. These expressions, which assume that probe reports represent an independent random sample from the traffic stream, indicate that as the number of probe reports in a period increases, the sample mean approaches the population mean.

The same assumption was used by other researchers in determining the required level of market penetration or number of probe vehicles (Srinivasan and Jovanis, 1996; Turner and Holder, 1995; Boyce et al, 1991a, 1991b)

Sen et al (1997a, 1997b) examined field data collected from probe vehicles as part of the ADVANCE project. On the basis of a statistical analysis of probe link travel times, they found that probe reports are not independent and therefore regardless of the sample size, the sample mean does not approach the population 
mean. They concluded that only "a small number of probe reports within a 5minute interval yields a standard error that is not substantially improved by making the number of probes much larger".

The conclusions reached by Van Aerde et al. (1993) and Sen et al. (1997) appear to be contradictory. This paper will show that both results are indeed correct but each is appropriate only for specific traffic and sampling conditions, and neither result can be held as a generalization for all traffic network conditions.

The remainder of this paper consists of three components. In the next section it is shown from fundamental queuing theory, that bias in the probe sample leads to a sample mean that does not asymptotically approach the population mean, regardless of the sample size. Following this, simulation data are used to illustrate the impact of sample bias on a signalized arterial. Finally conclusions are made regarding the importance of these findings for the design of probe based ATIS and ATMS.

\section{Analytical Estimation of the Expected Delay of Probe Vehicles:}

This section provides a theoretical estimate of the mean travel time experienced by a probe vehicle traversing a signalized arterial link. The objective is to show that the mean travel time of the probe vehicles (the sub-population from which samples are taken for estimation) may be different from the mean travel time of all the vehicles (population). The travel time that a vehicle experiences when traversing a signalized link consists of two components, namely the running time and the delay caused by the signal control. In the following theoretical derivation, we will assume that the mean running times of the probe vehicles and the general vehicles are the same and therefore we will focus on the difference in mean delay between probes and all vehicles. 


\section{Assumptions and Notations}

The delay that a probe vehicle experiences when it travels through a signalized approach depends on a number of factors including the arrival flow rate and distribution, signal timings and the time when the vehicle arrives at the approach. In a real application environment, many of these factors are random variables. As a result, the travel time reported by probe vehicles would likely be subject to large variation. For the purpose of illustrating the effect of sample bias, we will consider an idealized intersection approach consisting of a single through lane controlled by a signal with known timings. The approach has unlimited space for queuing and has a constant saturation flow rate. Furthermore, it is assumed that vehicle arrivals at the approach are uniformly distributed and consist only of passenger car units (pcu). The other notations are described as follows:

\section{Signal Timing Parameters}

$$
\begin{array}{ll}
c_{y} & =\text { cycle time (seconds) } \\
g & =\text { effective green interval (seconds) } \\
r & =\text { effective red interval (seconds) } \\
\lambda & =g / c_{y}
\end{array}
$$

\section{Arrival Flow}

$q_{g} \quad=$ average arrival flow rate during effective green interval ( $\mathrm{pcu} / \mathrm{second}$ ).

$q_{r}=$ average arrival flow rate during effective red interval (pcu/second).

$q \quad=$ average arrival flow rate during cycle time (pcu/second). Defined as

$$
q=\frac{q_{g} \cdot g+q_{r} \cdot r}{c_{y}}
$$

\section{Capacity}

$s$ = saturation flow rate (pcu/second).

$c_{a}=$ capacity (pcu/second), determined by $s \lambda$

$x=$ degree of saturation during the cycle time, defined as $q / c_{a}$

\section{Probe Vehicle Flow}

$P_{g} \quad=$ proportion of probe vehicles among all vehicles arriving during effective green interval. The probe arrival rate during effective green interval is therefore $q_{g} P_{g}$ (probe pcu/second) 
$P_{r}=$ proportion of probe vehicles among all vehicles arriving during effective red interval. The probe arrival rate during the red interval is therefore $q_{r} P_{r}$ (probe pcu/second)

$q_{p}=$ average probe arrival flow rate during cycle time (pcu/seconds), defined as

$$
q_{p}=\frac{P_{g} \cdot q_{g} \cdot g_{e}+P_{r} \cdot q_{r} \cdot r}{c_{y}}
$$

$\phi=$ ratio of the proportion of probe vehicle arrivals during the effective green interval to the proportion of probe vehicle arrivals during the effective red interval, defined as $\phi=\frac{P_{g}}{P_{r}}$

\section{Distribution of Delay}

Figure 1 illustrates the arrival rate for all vehicles and for probe vehicles only. Consider the case that a probe vehicle is randomly sampled from all probe vehicles arriving at the approach during the cycle time. The arrival time of the sampled probe vehicle, noted as $T$, would be a step-wise uniformly distributed random variable and its distribution can be described by Equation 3 .

$$
f_{T}(t)= \begin{cases}\frac{P_{r} \cdot q_{r}}{q_{p} \cdot c_{y}} & \text { if } 0<t \leq r \\ \frac{P_{g} \cdot q_{g}}{q_{p} \cdot c_{y}} & \text { if } r<t \leq c_{y}\end{cases}
$$

It should be noted that if both $P_{r}$ and $P_{g}$ are replaced by 1.0 in Equation 3, the result is the PDF of the arrival time of a general vehicle sampled from all arriving vehicles (population).

For a vehicle arriving at the approach at a given time $t$, its delay (noted as $d$ for general vehicles and $d_{p}$ for probe vehicles) can be determined based on deterministic queuing theory (as shown in Figure 2). 


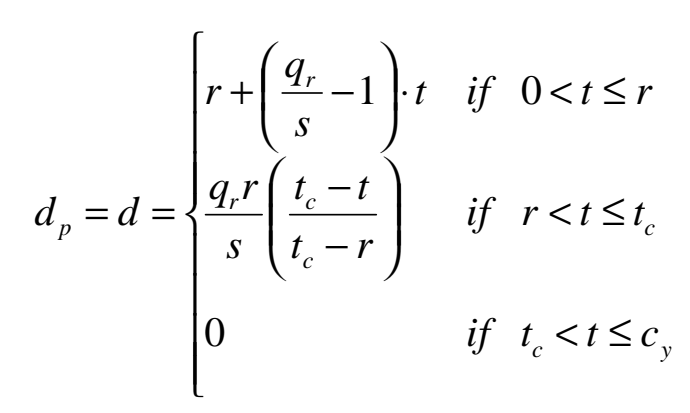

Where $t_{c}$ is the time when the queue is cleared and can be determined by

$$
t_{c}=r\left(1+\frac{q_{r}}{s-q_{g}}\right)
$$

In the case that a vehicle is randomly selected from the arriving flow, its delay would also be a random variable with its distribution depending on the distribution of its arrival time (Equation 3). Denote $D_{p}$ as the delay of a randomly selected probe vehicle, and $D$ as the delay of a randomly selected general vehicle. The following section discusses the derivation of the distribution of $D_{p}$. Note that the distribution of $D$ can be easily obtained from the distribution of $D_{p}$ by setting $P_{r}=P_{g}=1.0$

First, the sampled vehicle may experience no delay and the probability of this outcome is equal to the probability that the vehicle arrives during the time interval $\left[t_{c}, c_{y}\right]$, which can be determined from Equation 3:

$$
\begin{aligned}
P\left(D_{p}=0\right) & =P\left(t_{c}<T<c_{y}\right) \\
& =\left(c_{y}-t_{c}\right) \cdot \frac{P_{g} \cdot q_{g}}{q_{p} \cdot c_{y}}=\frac{P_{g} \cdot q_{g}}{q_{p} \cdot c_{y}}\left(g_{e}-\frac{q_{r} \cdot r}{s-q_{g}}\right)
\end{aligned}
$$


Second, as shown in Equation 4, the vehicle may experience a delay that decreases linearly from $\frac{q_{r} r}{s}$ to zero when the arrival time increases from $r$ to $t_{c}$. The probability that the vehicle would experience a delay greater than zero and less than or equal to $\frac{q_{r} r}{s}$ is therefore equal to the probability that the vehicle arrives during the time interval $\left[r, t_{c}\right]$ (Equation 7).

$$
\begin{aligned}
P\left(0<D_{p} \leq \frac{q_{r} \cdot r}{s}\right) & =P\left(r<T \leq t_{c}\right) \\
& =\left(t_{c}-r\right) \cdot \frac{P_{g} \cdot q_{g}}{q_{p} \cdot c_{y}} \\
& =\frac{q_{r} \cdot r}{\left(s-q_{g}\right)} \cdot \frac{P_{g} \cdot q_{g}}{q_{p} \cdot c_{y}}
\end{aligned}
$$

Since the arrival time is uniformly distributed over the arrival time interval $\left[r, t_{c}\right]$, delay should also be uniformly distributed with its PDF as shown in Equation 8.

$$
\begin{aligned}
f_{D_{p}}\left(d_{p}\right) & =\frac{P\left(0<D_{p}<\frac{q_{r} \cdot r}{s}\right)}{\left(\frac{q_{r} \cdot r}{s}-0\right)} \\
& =\frac{P_{g} \cdot q_{g} \cdot s}{q_{p} \cdot c_{y} \cdot\left(s-q_{g}\right)} \quad\left(0<d_{p} \leq \frac{q_{r} \cdot r}{c_{a}}\right)
\end{aligned}
$$

Similarly, the probability that the vehicle would experience a delay greater than $\frac{q_{r} r}{s}$ and less than or equal to $r$ can be determined based on Equation 4. 


$$
\begin{aligned}
P\left(\frac{q_{r} \cdot r}{s}<D_{p}<r\right) & =P(0<T<r) \\
& =(r-0) \cdot \frac{P_{r} \cdot q_{r}}{q_{p} \cdot c_{y}} \\
& =\frac{P_{r} \cdot q_{r} \cdot r}{q_{p} \cdot c_{y}}
\end{aligned}
$$

The PDF of the delay within this regime is

$$
\begin{aligned}
f_{D_{p}}\left(d_{p}\right) & =\frac{P\left(\frac{q_{r} \cdot r}{s}<d_{p}<r\right)}{\left(r-\frac{q_{r} \cdot r}{s}\right)} & \\
& =\frac{P_{r} \cdot q_{r} \cdot s}{q_{p} \cdot c_{y} \cdot\left(s-q_{r}\right)} & \left(\frac{q_{r} \cdot r}{s}<d_{p} \leq r\right)
\end{aligned}
$$

In summary, the delay of sampled probe vehicle is a mixed discrete and continuous random variable with its PMF represented by Equation 6, 7 and 9 and PDF represented by Equations 8 and 10.

\section{Mean Delay of Probe Vehicles}

With the given distribution functions, the mean delay of probe vehicles, $\mathrm{E}\left[D_{p}\right]$, can be obtained through the following mathematical expectations:

$$
E\left[D_{p}\right]=0 \cdot P\left(D_{p}=0\right)+\int_{0}^{\frac{q_{r}}{s} r} f_{D_{p}}(x) x d x+\int_{\frac{q_{r}}{s} r}^{r} f_{D_{p}}(x) x d x
$$

Based on Equations 8 and 10, Equation 11 can be rewritten as,

$$
E\left[D_{p}\right]=\frac{s \cdot r^{2}}{2 \cdot q_{p} \cdot c_{y}}\left[\frac{P_{r} \cdot q_{r}}{s-q_{r}}\left(1-\frac{q_{r}{ }^{2}}{s^{2}}\right)+\frac{P_{g} q_{g}}{s-q_{g}}\left(\frac{q_{r}{ }^{2}}{s^{2}}\right)\right]
$$


Consider a more idealized situation where no platoon progression exists and the arrival flow rate during the effective green interval is the same as the arrival rate during the effective red interval (i.e. $q_{g}=q_{r}=q$ ). Then Equation 12 can be simplified to Equation 13.

$$
\begin{aligned}
E\left[D_{p}\right] & =\frac{r^{2}}{2 \cdot c_{y} \cdot\left(1-\frac{q}{s}\right)} \cdot \frac{1+x^{2} \lambda^{2}(\phi-1)}{1+\lambda(\phi-1)} \\
& =E[D] \cdot \frac{1+x^{2} \lambda^{2}(\phi-1)}{1+\lambda(\phi-1)} \quad(x \leq 1.0)
\end{aligned}
$$

From Equation 13, it can be observed that if the probe arrival ratio $(\phi)$ is equal to 1 (i.e. a randomly selected probe can be considered as a general vehicle), the resulting equation is the well-known expression for uniform delay for all vehicles (see for example Hurdle, 1984; Teply et al, 1995). This implies that the expected delay of the probes is equal to the expected delay of all vehicles (population).

However, when the proportion of probe arrivals during the green interval $\left(P_{g}\right)$ is not equal to the proportion of probe vehicle arrivals during the red interval $\left(P_{r}\right)$ then $\phi \neq 1$ and $\mathrm{E}\left[D_{p}\right]$ is no longer equal to the expected mean of the population $(\mathrm{E}[D])$. Physically this means that if a disproportionate number of probe reports are received from probes that arrive during the green or red interval, then the sample is no longer random, but is biased. Consequently, the average delay computed from the probe reports is also biased and will asymptotically approach the probe mean $\left(\mathrm{E}\left[D_{p}\right]\right)$ but not the population mean $(\mathrm{E}[D])$ even if the number of probe reports is very large.

The theoretical analysis in this section has examined an idealized signalized intersection in which the delay associated with only a single link departure movement has been considered. However, the results are equally applicable to 
intersections with more than one outbound movement (e.g. left-turn, through, and right-turn movements), if each outbound movement is considered individually. The next section examines the potential sources of bias that are likely to be encountered in field conditions, and addresses the issue of multiple outbound movements.

\section{Sources of Sample Bias}

An important practical issue then, is to determine under what conditions the probe sample can be considered a biased sample. The previous section has shown, based on a theoretical derivation, that for an idealized intersection arrival time bias in the sample will lead to a systematic bias in the estimate of the population mean. If we consider that the objective is to estimate the population mean link travel time, where the population consists of all vehicles traversing the link regardless of the movement used to exit the link, then for a typical signalized link, the sample of probe reports can become biased in two ways:

1. First, the distribution of link entry times for probes may differ from the population. The time at which a vehicle enters the upstream end of a link depends largely on the turning movement required to access the link and the traffic controls impacting that movement. For example, consider the network illustrated in Figure 4 in which the intersection bounding the upstream end of the link is signalized and consists of 4 approaches. Vehicles accessing the link for which the delay is being measured do so via one of three possible movements, namely a left turn from the cross street; a right turn from the cross street; or a through movement from the main-street. Each of these movements is controlled by the upstream traffic signal and by gap acceptance behavior for opposed movements (i.e. left turn on green, and right turn on red). As illustrated in Figure 4, the distribution of link entry times is different for each of the three 
movements. Consequently, if the proportion of probe vehicles varies with each movement, then it also follows that the probe link entry time distribution will be different from that associated with the population. Since delay at the downstream intersection is a function of arrival time, a bias in arrival time will also result in a bias in delay.

2. The second cause of bias is associated with the movement used to exit the link (i.e. left turn; through, or right turn) at the downstream intersection. Typically, each movement experiences a different average delay. If the proportion of probe vehicles varies with each exit movement, then the sample is biased as a result of over or under sampling an exit movement that experiences a delay that is greater than or less than the population average.

Consequently, for a link bounded by a 4-leg intersection at the upstream and downstream ends, there exist 9 distinct sub-populations (one associated with each combination of entry and exit movement), each with its own set of travel time characteristics. If the proportion of probes varies across these 9 sub-populations then a bias will result.

\section{Effect of Sample Bias within a Sample Network}

To illustrate the potential magnitude of the sample bias, a simple linear network was modeled using the INTEGRATION traffic simulation model (Van Aerde et al, 1996). The network, illustrated in Figure 5, consists of a single arterial roadway that is intersected by two cross streets. Each intersection is controlled by a fixed-time signal $\left(c_{y}=120\right.$ seconds; $g=75$ seconds for the arterial; $g=37$ seconds for the cross street; offset $=0$ seconds).

The network is modeled for 1 hour with constant (time invariant) demands. Vehicles are generated at all origin zones with negative exponentially distributed 
headways. The O-D traffic demands between each of the 6 zones are provided in Table 1. The application of these demands to the network results in the intersection approaches experiencing $\mathrm{V} / \mathrm{C}$ ratios ranging from approximately 0.3 to 0.75 . Consequently, all of the intersection approaches operate in an undersaturated mode.

The time to traverse each link segment, the unique vehicle ID number, the time when the vehicle departed the link (i.e. time of probe report), and the vehicle's origin and destination were recorded for each vehicle. This log represented the travel times experienced by the entire vehicle population.

Three probe sampling scenarios were defined as follows:

1. Unbiased: all O-D pairs were sampled at the same level of market penetration (LMP).

2. Biased 1-6: only vehicles travelling between origin 1 and destination 6 were sampled.

3. Biased 2-4: only vehicles travelling between origin 2 and destination 4 were sampled.

For each sampling scenario, simulations were conducted for 22 levels of market penetration (i.e. 0.0 to 0.3 in 0.02 increments and 0.4 to 1.0 in 0.1 increments). For each test, probe reports were aggregated into 5-minute periods (12 periods in total). The mean segment travel time computed on the basis of the probe reports, and the number of probe reports within each period were recorded for each test. The results are examined for vehicles traveling eastbound on link segments 1, 2, and 3 only (Figure 5).

Segment 1 represents a link that is not affected by in traffic signals at either its upstream or downstream and for which only a single entry movement and exit movement is possible. Therefore, a biased sample (based on over or under sampling a sub-population) is not possible. Furthermore, since the link is not 
controlled by a signal, the link travel times are not expected to exhibit a great amount of variation.

Segment 2 represents a link for which bias can only arise as a result of over or under sampling the downstream exit movements.

Segment 3, having a signalized intersection at both the upstream and downstream boundaries, is susceptible to bias as a result of over or under sampling any of the 9 possible combinations of entry and exit movements.

Figure 6 illustrates the mean travel time for segment 1 estimated from probe reports received during each 5-minute period, as a function of the number of probe reports. The 95\% confidence limits (C.L.) of the estimated mean segment travel time are also illustrated. These confidence limits are computed from Equation 14 using the entire vehicle population under the assumption that probe reports represent a randomly selected sample from a single infinite population. For segment 1 , there is no opportunity for bias by over or under sampling a specific entry or exit movement, so this assumption is valid.

$$
\text { C.L. of } \bar{x}=\bar{x} \pm z\left(\frac{\operatorname{VAR}\left(x_{i}\right)}{n}\right)
$$

Where $\bar{x}$ is the mean segment travel time computed from the entire population of vehicles traversing the segment during the 1-hour simulation; $\operatorname{VAR}\left(x_{i}\right)$ is the variance of the individual vehicle segment travel times about $\bar{x} ; n$ is the number of probe reports received during the time interval; and $z$ is the normal standard deviate associated with the confidence limits (i.e. $z=1.96$ for a confidence limit of $95 \%)$.

Three observations can be made from Figure 6. First, the mean travel times are all within a very small range of between 23 and 28 seconds. This is expected, since no signal impacts this link. Second, the 5-minute average travel times are 
distributed between the confidence limits with no apparent bias. Third, the confidence limits of the sample mean show an initial rapid reduction in the error of the estimate as the number of probe reports increases. Furthermore, the error tends to zero as the number of reports approaches infinity.

Figure 7 illustrates the mean 5-minute travel times obtained from probe reports for biased and unbiased samples. From these results it is evident that having a biased sample results in mean travel time estimates that do not represent the travel time experience of the population. In particular, when sampling from only those vehicles travelling from origin 1 to destination 6 , the resulting sample mean travel times are much larger than the population mean travel times. This is expected as this sample of vehicles is required to make a left turn at the downstream end of segment 2 , and consequently experience much greater delay than the population of vehicles traversing segment 2 .

Figure 8 illustrates the effect of bias in the arrival time distribution between probe vehicles and general vehicles traversing segment 3. Mean travel times from two samples are presented. The unbiased sample reflects the experience of the true population. The biased sample only reflects vehicles that are travelling between origin 2 and destination 4 . From the results in Figure 8 it is evident that the biased sample travel times consistently underestimate the population travel time.

While the biases presented in this example network can be considered to be extreme, they do serve to illustrate the potential extent of the problem. Furthermore, the results from Sen et al. (1997a) discussed earlier in this paper, are based on field data in which all probe vehicles traversed a set of links by using the same link entry and link exit movements. Thus, the sample used in their analysis has a level of bias that is similar to that associated with the examples provided in this paper. The findings described within this paper demonstrate that the 
conclusions of Sen et al, that "a small number of probe reports within a 5-minute interval yields a standard error that is not substantially improved by making the number of probes much larger", should not be generalized!

\section{Conclusions and Recommendations}

Previous research in the literature has provided seemingly contradictory conclusions regarding the accuracy of mean link travel times estimated from probe vehicle reports. The apparent disagreement between these results gives rise to confusion among practitioners and may lead to inappropriate ATIS design decisions.

This paper has examined the issue of the accuracy of mean travel times as estimated from probe vehicles. More specifically, this paper has shown that under conditions when the probe vehicles represent a biased sample, the sample mean does not approach the population mean. It has also been shown that for a typical link, that is bounded at both the upstream and downstream ends by a signalized intersection, the population of vehicles can be divided into 9 sub-populations, each associated with a unique turning movement combination for entering and exiting the link. If the proportion of probe vehicles varies between these 9 subpopulations, the probe reports represent a biased sample and error associated with the sample mean may remain quite large, even when the sample size is large. However, if the proportion of probe vehicles is nearly constant across all of the 9 sub-populations, then the sample is unbiased, and the probe reports can be considered to represent independent samples from the population. In this case, standard sampling theory holds, and the error of the sample mean decreases as the sample size increases.

Thus, the identification of sample bias enables the apparently inconsistent results of previous research reported in the literature, to be more clearly 
interpreted. Specifically, the work of Sen et al. (1997a) can be seen as applicable to scenarios in which the probe reports constitute a biased sample of the population. Conversely, the work of Van Aerde et al. (1993) is applicable when the probe reports are an unbiased sample of the population.

It has been shown that the degree to which the probe reports represent a biased sample is critical in assessing the reliability of the sample mean as an estimate of the population mean. Therefore, it is recommended that further research focus on developing methods that can be applied under field conditions to quantify the degree of bias associated with a sample of probe reports. Furthermore, if this bias can be quantified, it is recommended that methods be developed by which the impact of this bias can be reduced or eliminated in order to provide more accurate estimates of the population mean travel time.

\section{Acknowledgements}

The authors gratefully acknowledge the financial support provided by the Natural Sciences and Engineering Research Council of Canada.

\section{References}

Boyce, D., Kirson, A. , and Schofer J. (1991a). "Design and implementation of ADVANCE: The Illinois dynamic navigation and route guidance program." Proceedings of the Vehicle Navigation and Information Systems Conference, Vol. 1. Dearborn, Mich. Society of Automotive Engineers, Warrendale, PA. pp. 415426.

Boyce, D., Hicks, J. and Sen A. K. (1991b). "In-vehicle navigation system requirements for monitoring link travel times in a dynamic route guidance system." Presented at the $70^{\text {th }}$ Annual Meeting of the Transportation Research Board, Washington D.C. 
Hurdle, V. F. (1984). "Signalized intersection delay models - a primer for the uninitiated." Transportation Research Record 971, Transportation Research Board, Washington, D.C. pp. 96 - 105.

Sen A., Thakuriah, P., Zhu, X., and Karr, A. (1997a). "Frequency of probe reports and variance of travel time estimates." Journal of Transportation Engineering Vol. 123 No. 4. ASCE, pp. 290 - 297.

Sen, A., Soot, S., Ligas, J., and Tian, X. (1997b). Arterial Link Travel Time Estimation: Probes, Detectors and Assignment-type Models. Presented at the 76th Annual Meeting of the Transportation Research Board, Washington, D. C. Preprint No. 970943.

Srinivasan, K. K. and Jovanis, P. P. (1996). "Oversaturation Delay Estimates with Consideration of Peaking." Transportation Research Record 1537, Transportation Research Board, Washington D. C. pp. 15-22.

Teply, S., Allingham, D. I., Richardson, D.B., and Stephenson, B.W. (1995). Canadian Capacity Guide for Signalized Intersections. Institute of Transportation Engineers, District 7- Canada.

Turner, M. S. and Holdener, D. J. (1995). "Probe Vehicle Sample Sizes for Real-Time Information: The Houston Experience." Proceedings of Vehicle Navigation and Information Systems (VNIS) Conference, July 30 August 2, 1995 , Seattle, Washington. IEEE. pp. 3-9

Van Aerde, M., Hellinga, B., Yu, L., and Rakha, H. (1993). "Vehicle Probes as Real-Time ATMS Sources of Dynamic O-D and Travel Time Data." Large Urban Systems - Proceedings of the ATMS Conference held in St. Petersburg, Florida. pp. 207- 230

Van Aerde, M., Hellinga, B., Baker, M., and Rakha, H. (1996). "INTEGRATION: Overview of Simulation Features." Paper presented at $75^{\text {th }}$ 
Annual Meeting of the Transportation Research Board, Transportation Research Board, Washington, D.C. 


\section{LIST OF TABLES}

Table 1. O-D traffic demands for test network (vph) 


\begin{tabular}{|c|c|c|c|c|c|c|c|}
\hline \multirow{2}{*}{$\begin{array}{l}\text { Origin } \\
\text { Zone }\end{array}$} & \multicolumn{6}{|c|}{ Destination Zone } & \multirow[t]{2}{*}{ Total } \\
\hline & 1 & 2 & 3 & 4 & 5 & 6 & \\
\hline 1 & & 300 & 162 & 808 & 81 & 100 & 1450 \\
\hline 2 & 75 & & 31 & 154 & 15 & 600 & 875 \\
\hline 3 & 38 & 5 & & 100 & 200 & 7 & 350 \\
\hline 4 & 920 & 120 & 150 & & 200 & 160 & 1550 \\
\hline 5 & 192 & 25 & 500 & 200 & & 33 & 950 \\
\hline 6 & 250 & 400 & 8 & 38 & 4 & & 700 \\
\hline Total & 1475 & 850 & 850 & 1300 & 500 & 900 & 5875 \\
\hline
\end{tabular}

Table 1: O-D traffic demands for test network (vph) 


\section{LIST OF FIGURES}

FIG. 1. Illustration of arrival rates for probe vehicles and all vehicles during the red and green intervals

FIG. 2. Deterministic queuing diagram

FIG. 3. Uniform queue delay as a function of vehicle arrival time

FIG. 4. Arrival time bias as a function of link entry movement

FIG. 5.Arterial test network configuration

FIG. 6. Mean 5-minute travel times and 95\% confidence limits for unbiased sample (Segment 1)

FIG. 7. Mean 5-minute travel times from unbiased and biased samples as a function of number of probe reports (Segment 2)

FIG. 8. Mean 5-minute travel times from unbiased and biased samples as a function of the number of probe reports (Segment 3) 


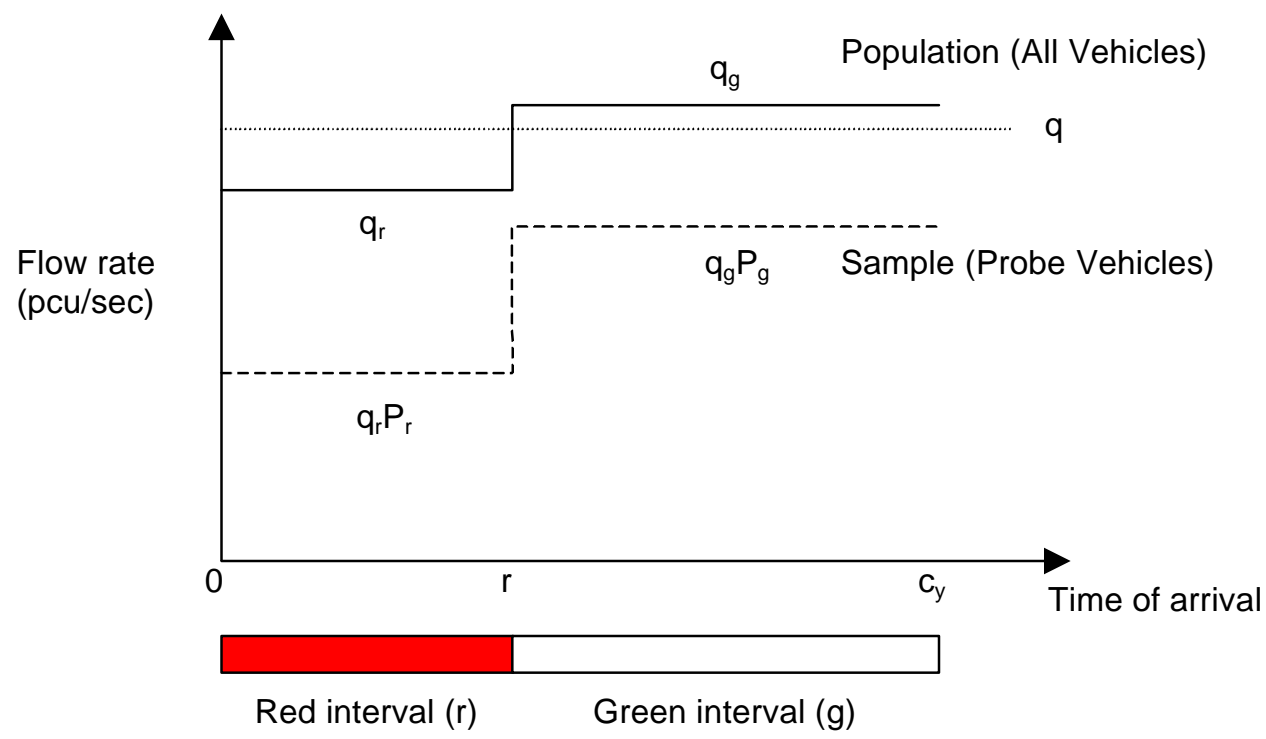

Figure 1: Illustration of arrival rates for probe vehicles and all vehicles during the red and green intervals 


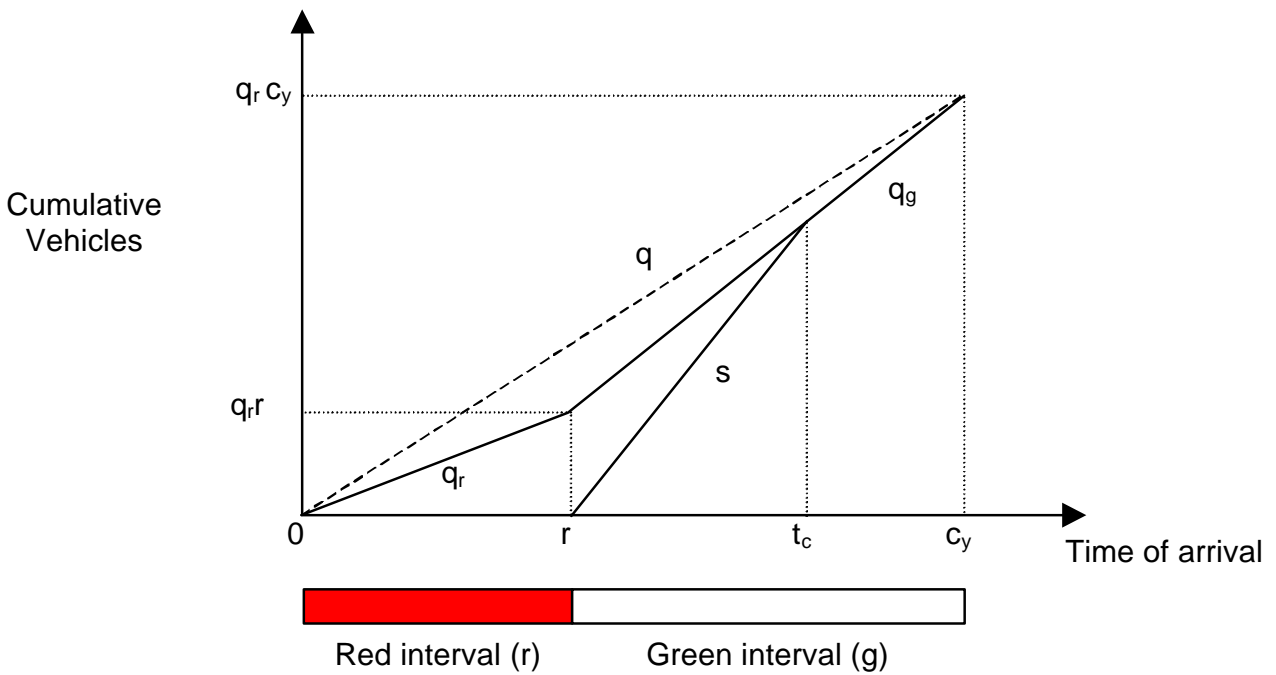

Figure 2: Deterministic queuing diagram 


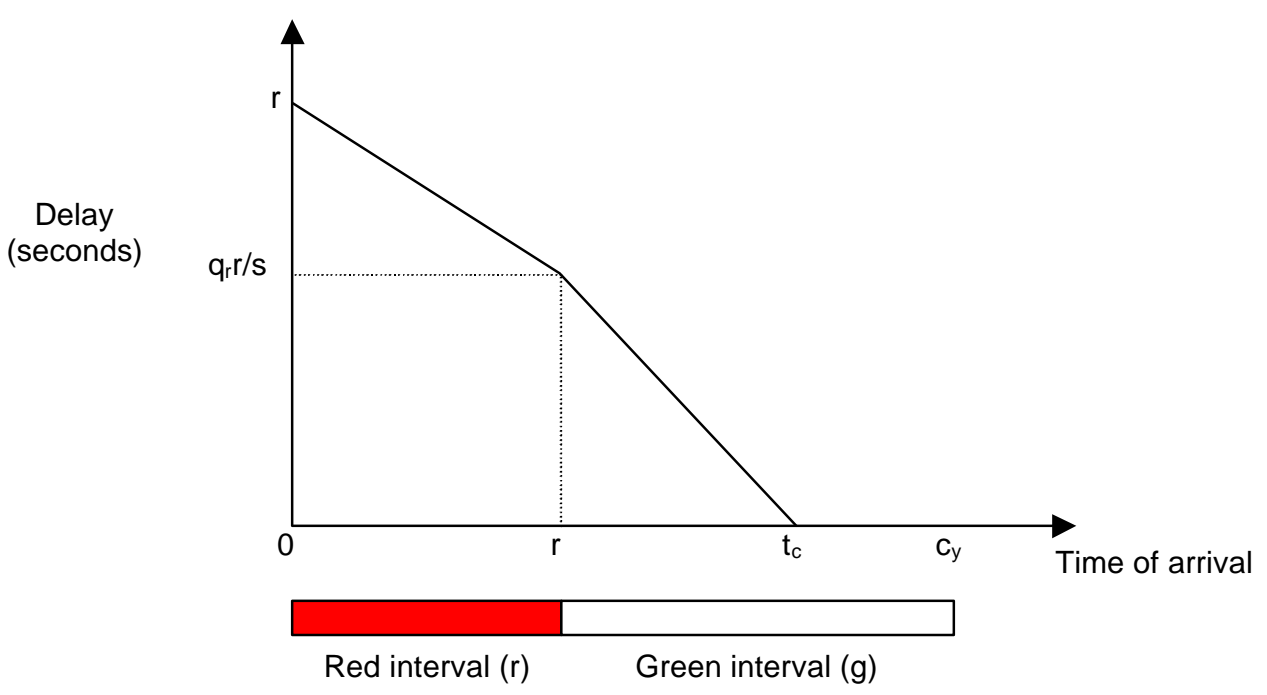

Figure 3: Uniform queue delay as a function of vehicle arrival time 

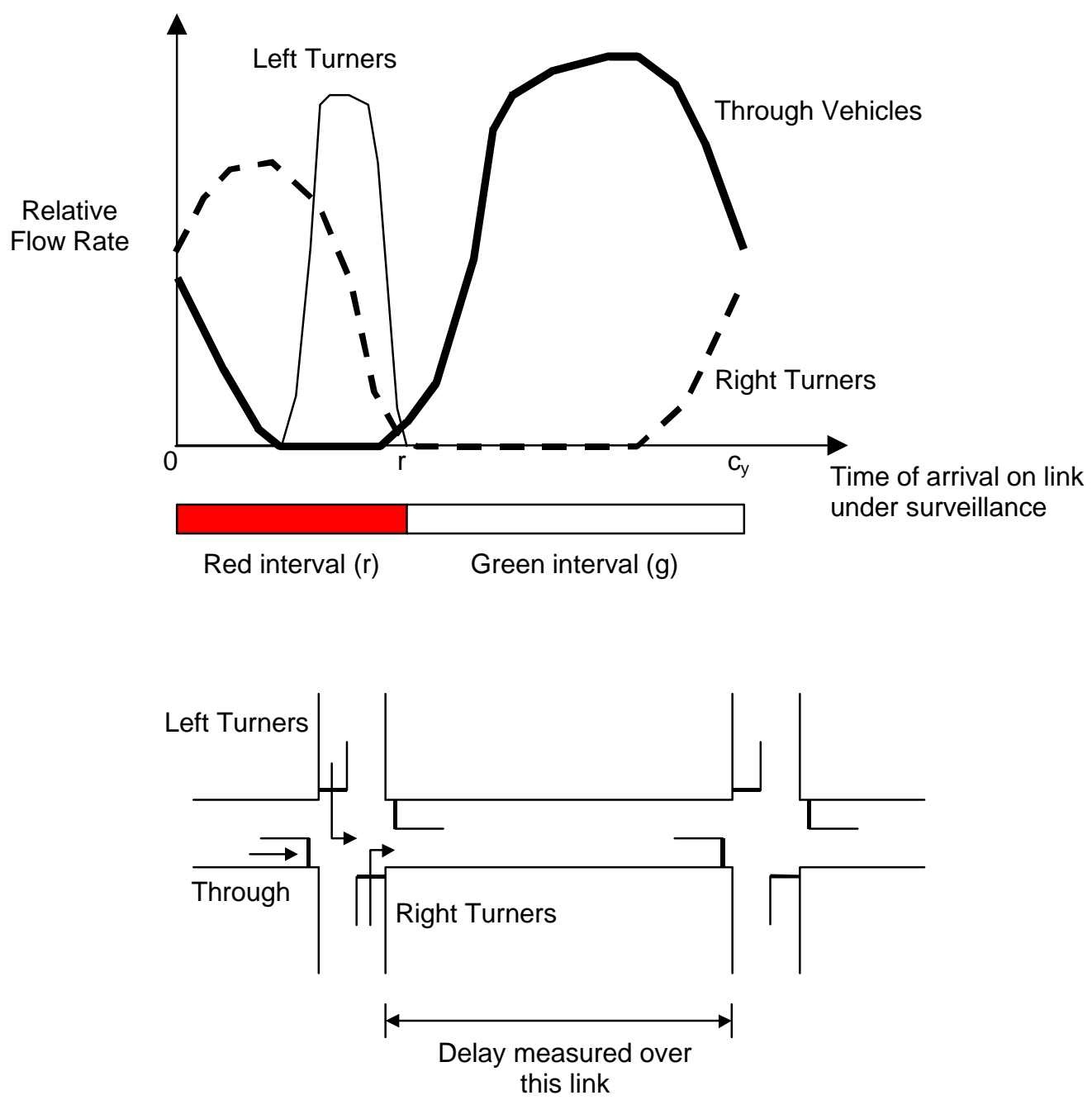

Figure 4: Arrival time bias as a function of link entry movement 


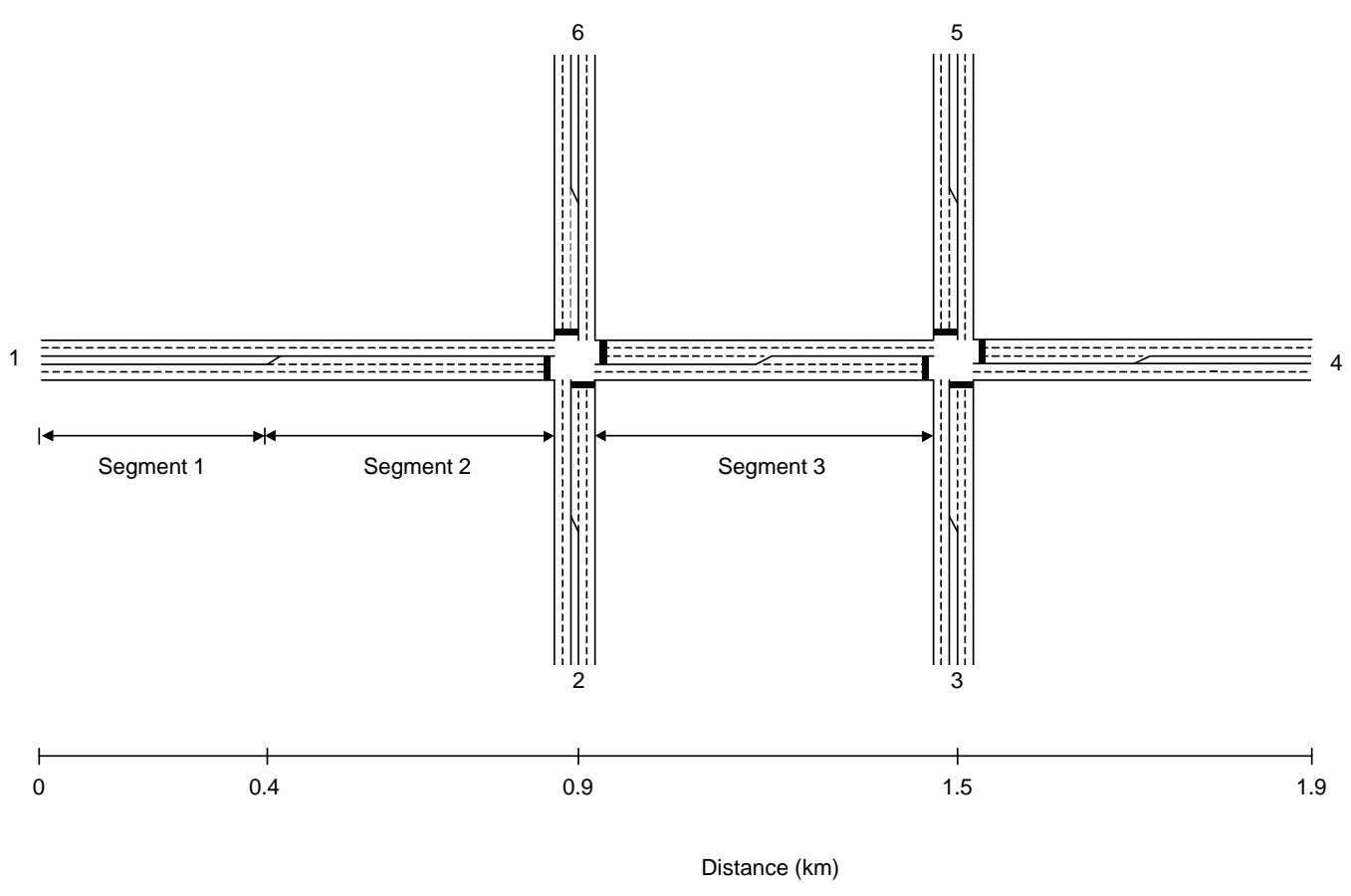

Figure 5: Arterial test network configuration 


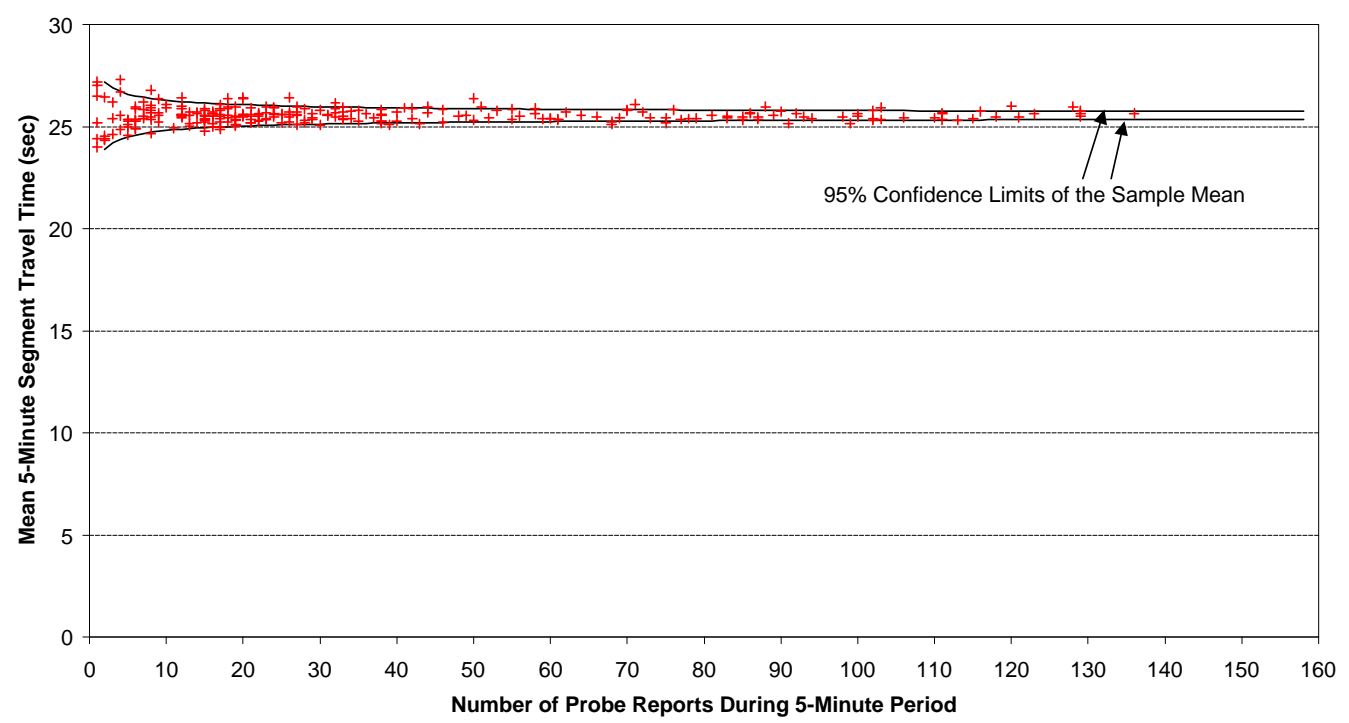

Figure 6: Mean 5-minute travel times and 95\% confidence limits for unbiased sample (Segment 1) 


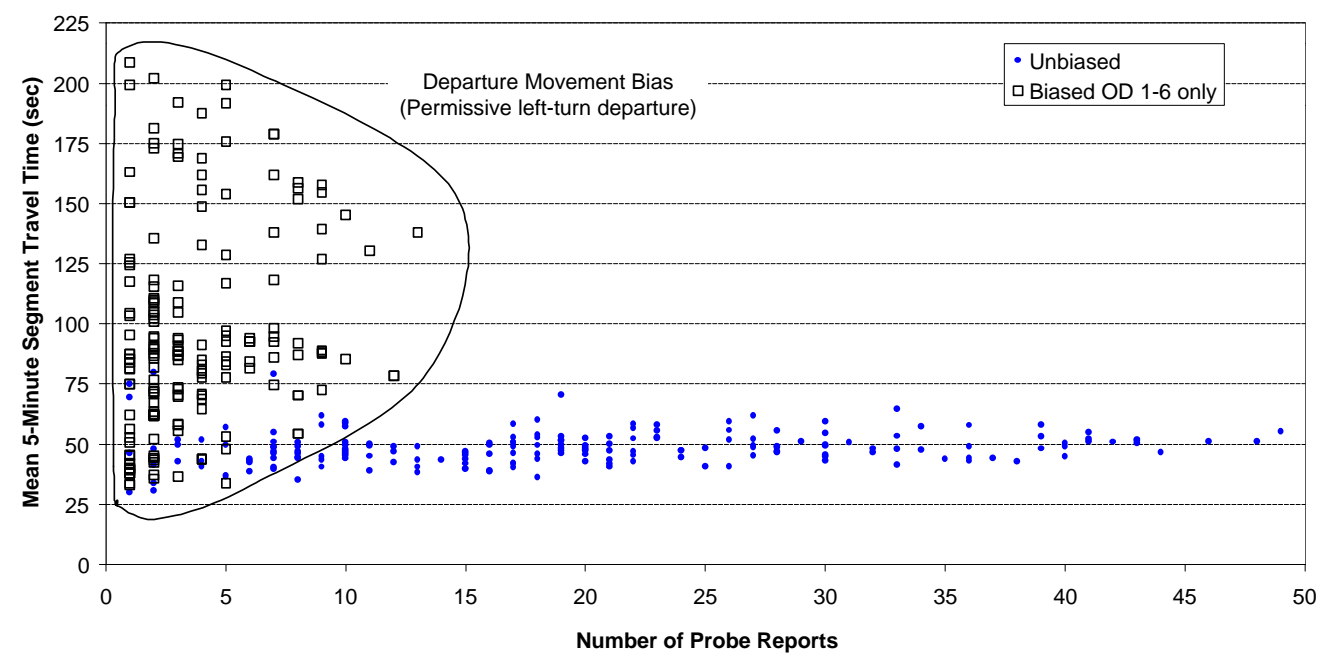

Figure 7: Mean 5-minute travel times from unbiased and biased samples as a function of number of probe reports (Segment 2) 


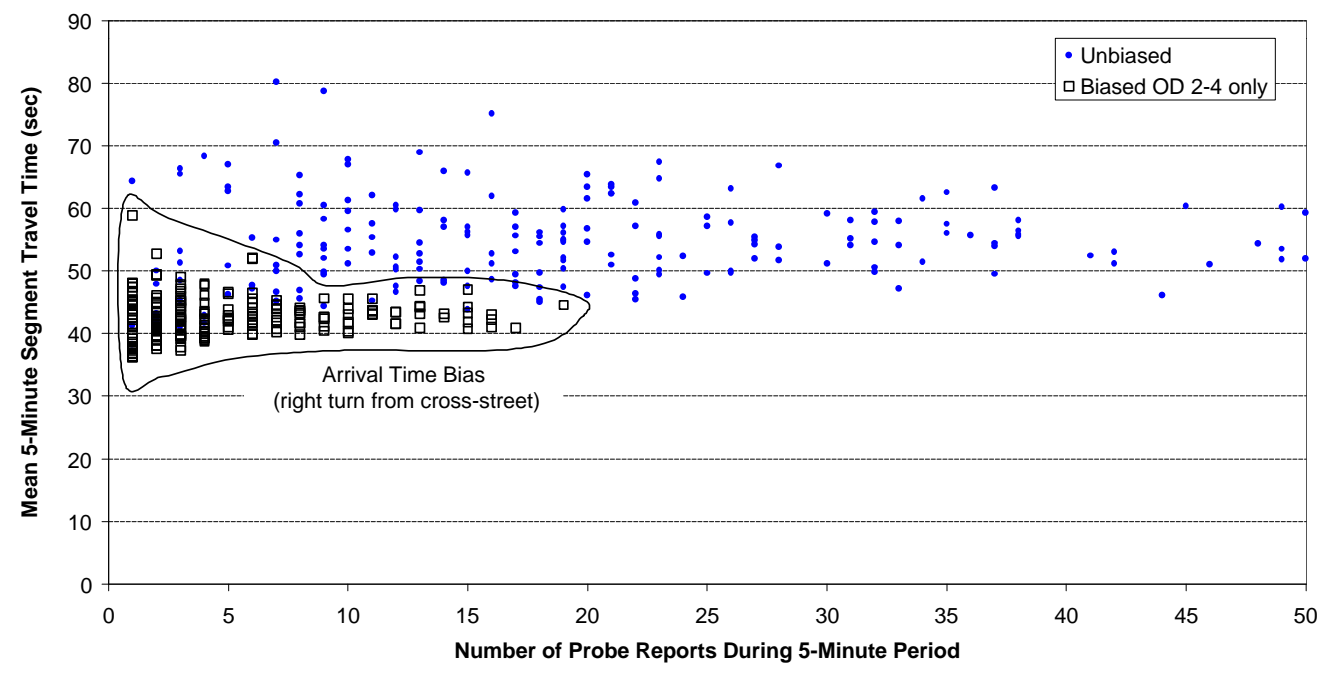

Figure 8: Mean 5-minute travel times from unbiased and biased samples as a function of the number of probe reports (Segment 3 ). 